\section{Moderate exercise is safe and improves fitness in patients with Becker muscular dystrophy}

Previous studies in a mouse model of dystrophinopathy have indicated that exercise might induce muscle damage and accelerate disease progression in this setting. On the basis of these findings and clinical experience, individuals with Becker muscular dystrophy (BMD) are advised to avoid excess physical exertion. Sveen et al. have performed the first study to date that has thoroughly investigated the effects of moderate exercise in patients with BMD.

This study enrolled 11 men with BMD and 7 healthy, sedentary controls. All participants took part in 50 training sessions over a period of 12 weeks. Training comprised 30-minute sessions on stationary cycle ergometer at a heart rate corresponding to $65 \%$ of maximum oxygen uptake.

The 12-week training regimen improved fitness in patients with $\mathrm{BMD}(P<0.005)$; this effect was sustained in the six patients who continued training for 1 year. All participants showed an increase in strength in the muscle groups involved in cycling. There was no increase in levels of plasma creatine kinase-an indication of muscle damage-in the patients with BMD, nor were there any significant changes in muscle morphology. The majority of patients with BMD reported an improvement in physical endurance, leg muscle strength and walking distance after the 12-week training period.

The authors conclude that moderate exercise does not cause muscle damage and is a safe and effective way to produce a long-term increase in fitness in patients with BMD.

Original article Sveen ML et al. (2008) Endurance training improves fitness and strength in patients with Becker muscular dystrophy. Brain [doi:10.1093/brain/awn189]

\section{Sumatriptan reduces somatic and visceral pain in mice}

Triptan drugs are commonly prescribed for the treatment of migraine and are presumed to act on a subset of 5-hydroxytryptamine receptors at pain sensory neurons in the brain. However, triptan receptors are not exclusively located in the brain; they are found on pain sensory neurons throughout the body. Nikai and colleagues, therefore, hypothesized that triptans might also reduce noncranial, somatic and visceral pain.

The team assessed the effects of sumatriptan, administered either by subcutaneous injection (systemic) or intrathecal injection (localized in the spine), in mouse behavioral models of chronic and acute pain. Neither subcutaneous nor intrathecal sumatriptan had any effect on acute pain. For a model of persistent pain, the mice were injected with dilute formalin into the hind paw. Intrathecal sumatriptan had a modest effect on pain behaviors in the early phase of the formalin test, but the drug profoundly reduced pain behaviors in the second phase. Indeed, intrathecal administration was observed to completely reverse the mechanical and thermal hypersensitivity in another model of persistent pain. Subcutaneous sumatriptan, on the other hand, offered no analgesia. Intrathecal sumatriptan was superior to subcutaneous injection in a model of visceral pain (intraperitoneal acetic acid), conferring a marked reduction $(\sim 80 \%)$ in observed pain. Neither method of delivery was found to be effective in a model of nerve injury, suggesting that not all chronic pain conditions would be amenable to sumatriptan.

Nikai et al. conclude that intrathecal triptan administration could offer a new potential line of treatment for persistent somatic and visceral pain independent of headache.

Original article Nikai T et al. (2008) Profound reduction of somatic and visceral pain in mice by intrathecal administration of the anti-migraine drug, sumatriptan. Pain [doi:10.1016/j.pain.2008.06.002]

\section{Virtual reality identifies navigational defects in Alzheimer disease and cognitive aging}

Cushman et al. have previously characterized navigational impairments, which are indicative of cognitive aging or Alzheimer disease, by means of real-world navigational tests. Such tests can, however, be time-consuming and difficult to conduct. Cushman and colleagues, therefore, hypothesized that a virtual reality (VR) test could provide an alternative method of identifying navigational deficits.

The researchers studied performances in a real-world navigation test and a VR version simulating the same navigational environment 